\section{Stitch in time saves design}

\section{S. J. Judge}

THERE is currently considerable enthusiasm for 'neural network' models of brain function, but one issue that has not been resolved is how the structure of real brain circuits (which are by no means always the densely randomly interconnected networks favoured by the theoretical network modellers) can be incorporated into such models. Perhaps because its circuitry is relatively well known, the eye-movement system has attracted several attempts to marry the network approach to the particulars of real brain circuitry and function ${ }^{1-3}$. The latest of these attempts is to be found on page 159 of this issue ${ }^{4}$, where Lisberger and Sejnowski offer an intriguing model of the vestibulo-ocular reflex (VOR).

The VOR is the reflex that prevents the image of the world sliding over the retina during head turns. Head rotation is sensed by the semicircular canals in the inner ear, and that signal is used to produce eye movements that rotate each eye in its socket so as to keep eye angular velocity in space close to zero during head turns. Because even lowvelocity image movement impairs visual resolution, VOR gain (eye angular speed divided by head angular speed) should be kept close to unity.

\section{Brain circuitry}

There has been a longstanding dispute about the nature of the brain circuitry that adjusts VOR gain, with Ito ${ }^{5}$ championing the view that the VOR gain is adjusted by regulating the size of the vestibular signal flowing from the canals through a particular part of the cerebellum called the flocculus and back to the main pathway in the brainstem. The difficulty with accepting this argument is that by far the most thorough study of the behaviour of neurons in the flocculus, by Miles and his colleagues ${ }^{6}$, found that although the signals there did change when the VOR gain was manipulated (by experience in various optical devices that altered the relationship between head movement and its visual consequences) the signals changed in the wrong direction for Ito's hypothesis. An alternative model was therefore proposed $^{7}$ in which although gain does change at the connection between the incoming vestibular fibres and flocculus neurons, the key factor in altering VOR gain was regulation of the size of the vestibular signal flowing through the main brainstem pathway.

Lisberger and Sejnowski now present a very simple model which may be able to resolve the conflict between the two groups, and which also incorporates a principle that the authors suggest may have a more general application. This model is not itself a 'neural network' model, but a classic engineering model of a kind made familiar through the influential work of Robinson ${ }^{8}$. Lisberger and Sejnowski propose that, as well as gain changes in both the direct brainstem and cerebellar pathways, there is a third modifiable element, namely the speed of the dynamic response in the pathway carrying vestibular input to the cerebellum. Lest one gives the impression that Occam's razor is being neglected, let it

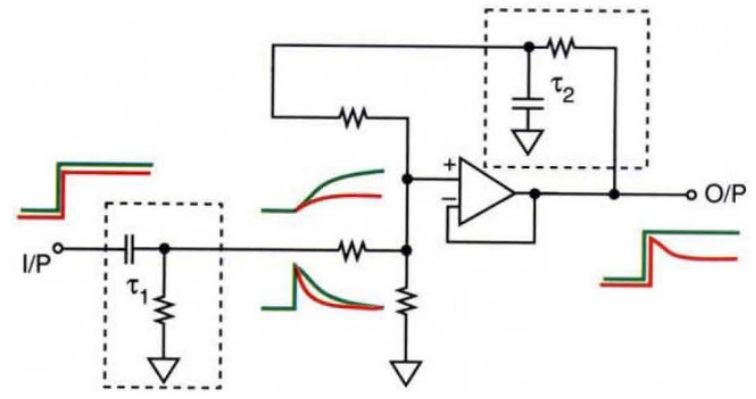

Electrical equivalent of Lisberger and Sejnowki's proposed neural circuit for the VOR. If the time constants $\tau_{1}$ of the input filter and $\tau_{2}$ of the filter in the positive feedback loop are equal the circuit will have unity gain, as shown in the waveforms in green. If $\tau_{1}$ is less that $\tau_{2}$ the circuit will respond to a step with the waveform shown in red and will have a d.c. gain of less than unity.

be said that the model requires the two gains to be matched, or it will become unstable at low frequencies. How the system manages to keep these gains matched is not explained.

To see how the dynamic element works, consider the electrical realization of Lisberger and Sejnowski's model (see figure). The vestibular input is filtered by a high-pass filter and summed with the output of a low-pass filter, whose input is itself the output of the whole circuit. (The unity gain amplifier is present only as a buffer.) The form of the output depends on the mismatch between the time constants in the two filters. If that of the first filter is smaller, then the response to a step input will be a step plus exponential slide in the same direction. If the time constant of the first filter is larger, then the response will be a step plus exponential slide back. In other words, the circuit converts a change in the dynamic properties of the input filter to a change in steady state gain. It is important to note that this brings about gain change without the adjustment of any gain 'knob' in the circuit, and it is this curious feature of the circuit that gives it the potential for reconciling the models of Ito and Miles et al. When the VOR gain is reduced, this is achieved by the combination of a modest reduction in gain in both the direct and cerebellar pathways, and a much more dramatic reduction in time constant of the cerebellar input. The latter allows the VOR gain to be reduced despite the fact that the gain of the connection between the vestibular input and the flocculus has changed in the 'wrong' direction. Why the gains should change at all is not explained.

\section{Hidden variable}

This idea is simple and ingenious, and of course one wishes it well. But it works by introducing a 'hidden variable' to account for otherwise contradictory data - although this is always a possible resolution of a contradiction, one wants to see direct evidence for the postulated change in dynamics. Although Lisberger and Pavelko ${ }^{9}$ have evidence that the dynamics of the eye-movement response to a head movement do change in a way compatible with the new model when VOR gain is changed, it is by no means certain that the neural input to the flocculus does alter its dynamics in the way supposed.

The circuit is of course a very odd one to the electrical engineer because its design feature is just the opposite of normal engineering practice, where one seeks to arrange the circuit so that its overall performance will be reasonably consistent despite incidental variations in the values of circuit components. This circuit has just the opposite property, in that it exploits the fact that small mismatches of components have drastic consequences. There is of course a price to be paid for such a design: the circuit is only quasi-stable. But, biologically, perhaps that is a price worth paying.

S. J. Judge is in the University Laboratory of Physiology, University of Oxford, Parks Road, Oxford OX1 3PT, UK.

1. Anastasio, T. J. \& Robinson, D. A. Neural Computn 1 230-241 (1989).

Robinson, D. A. A. Rev. Neurosci. 12, 33-45 (1984).

3. Lisberger, S. G. \& Sejnowski. T. J. Univ. California San Diego Inst. Neural Comput. Tech. Rep. 9201 (1992).

4. Lisberger, S. G. \& Sejnowski, T. J. Nature 360, 159-161 (1992).

5. Ito, M. A. Rev. Neurosci. 5, 275-296 (1982)

6. Miles, F. A. et al. J. Neurophysiol. 43, 1406-1493 (1980)

. Miles, F. A. \& Lisberger S. G. A. Rev. Neurosci. 4 273-299 (1981)

8. Robinson, D. A. A. Rev. Neurosci. 4, 463-503 (1981).

9. Lisberger, S. G. \& Pavelko, T. A. J. Neurosci. 6 346-354 (1986). 